\title{
Superpoderes contra el Dr. Discriminador. La mejora de la evaluación continua mediante la ludificación en el Máster en profesorado
}

\author{
Superpowers against Dr. Discriminator. Improving continuous \\ assessment by gamification in the Master's in teaching
}

\author{
José Maㅡ Falcó-Boudet \\ Universidad de Zaragoza \\ chema.falco@unizar.es \\ José Luis Huertas Talón \\ Universidad de Zaragoza \\ jhuertas@unizar.es
}

Recibido: 15/04/2018

Aceptado: 30/05/2018

Publicado: 29/06/2018

\section{RESUMEN}

La ludificación, esto es, motivar a las personas con las mecánicas de los juegos para que se involucren y participen con la finalidad de conseguir mejores resultados, también ha sido utilizada en contextos de Educación Superior. Con el objetivo de motivar la asistencia, la participación y la realización de las tareas de la evaluación continua en una asignatura del máster en profesorado, se implementó una dinámica de obtención de insignias ambientada en la consecución de superpoderes para luchar contra el Dr. Discriminador. La evaluación de los resultados se hizo mediante el análisis de las respuestas recogidas a través de un cuestionario y la comparación de los datos de asistencia y participación con los de cursos anteriores. Los resultados demuestran que, además de ser del agrado de los estudiantes, supuso un incentivo para realizar las actividades de evaluación continua.

\section{PALABRAS CLAVE}

Ludificación; Motivación; Formación del profesorado; Innovación docente.

\begin{abstract}
Gamification, motivating people with the mechanics of games to get involved and participate in order to achieve better results, is also being used in Higher Education. In order to motivate the attendance, participation and completion of the tasks of continuous assessment in a subject of the master's degree in teachers a dynamic was implemented to earn badges that symbolized the achievement of superpowers to fight against the Dr. Discriminator. The evaluation of the results was done by analyzing the answers gathered through a questionnaire and comparing the attendance and participation data with those of previous courses. The results show that students were satisfied with the experience and it was an incentive to carry out continuous assessment activities.
\end{abstract}

\section{KEYWORDS}

Gamification; Student Motivation; Teacher Education; Instructional Innovation.

\section{CITA RECOMENDADA}

Falcó-Boudet, J.M. y Huertas, J.L. (2018). Superpoderes contra el Dr. Discriminador. Una experiencia de ludificación en el Máster en profesorado. RIITE. Revista Interuniversitaria de Investigación en Tecnología Educativa, 4, 68-81. Doi: http://dx.doi.org/10.6018/riite/2018/327961 


\section{Principales aportaciones del artículo y futuras líneas de investigación:}

- La ludificación es una estrategia que también produce buenos resultados en la Educación Superior.

- La ludificación contribuye a mejorar la asistencia y el interés por la correcta realización de las tareas.

- Líneas de investigación: influencia de la ludificación en el ámbito educativo, pros y contras de su utilización.

\section{INTRODUCCIÓN}

"Superpoderes contra el Dr. Discriminador» es el resultado de ludificar las actividades de evaluación continua de la asignatura de Fundamentos de Diseño Instruccional y Metodologías de Aprendizaje de la especialidad de Tecnología e Informática del Máster en profesorado de la Universidad de Zaragoza que surge del análisis de las problemáticas observadas en cursos anteriores.

Al finalizar el curso 2016-17 los profesores responsables de esta experiencia de investigación-acción constatamos que:

- Se trata de una asignatura de difícil asimilación para alumnos con una formación científico-tecnológica.

- Experimentar en primera persona, como alumno, algunas de las metodologías de enseñanza-aprendizaje que forman parte del contenido curricular de la asignatura favorecería la interiorización de las mismas.

- Aunque la asistencia no es un requisito consideramos la conveniencia de premiar el esfuerzo de los alumnos que asistían a clase regularmente.

- La participación en las tareas de coevaluación era baja, con el perjuicio que ello supone para los alumnos a los que ningún compañero les valora su trabajo.

La ludificación es una estrategia que asegura la motivación del alumnado para completar las tareas (Sandusky, 2015), por lo que rediseñamos el currículo de la asignatura para mejorar en esos cuatro aspectos.

\section{ESTADO DEL ARTE}

Ludificación no es utilizar juegos, sino utilizar los elementos de propios de los juegos (puntos, recompensas, retroalimentación en tiempo real, autonomía de decisión, posibilidad de volver a empezar, ...) en otros entornos (Lee y Hammer, 2011). Desde que Pelling propuso en 2003 el término ${ }^{1}$ poco a poco se ha introducido cada vez en más ámbitos, desde la señalización del tráfico (la carita que nos sonríe si vamos a la velocidad adecuada por un punto determinado) a las campañas publicitarias (Samsung Nation, Nike + o ¡Agítalo! de Coca Cola), a las promociones del ámbito de la salud y el bienestar (FitBit, ¡Piérdelo!) o a las campañas de fidelización de sitios web (con cierto número de comentarios a productos se van subiendo niveles).

\subsection{Beneficios de la ludificación en educación}

En la actualidad se está implementando la ludificación en diversos programas educativos (Huang y Soman, 2013) porque la utilización de un marco simbólico (insignias, recompensas, ...) y estético (historia del juego, ambientación) mejora la experiencia de aprendizaje, la motivación y el compromiso del alumnado en la resolución de problemas (Deterding, Dixon,

\footnotetext{
${ }^{1}$ Hace referencia a la primera utilización del término inglés gamification
} 
Khaled, y Nacke, 2011). Las experiencias de ludificación en los estudios superiores se han desarrollado en diferentes contextos con buenos resultados, tal como se recoge en la Tabla 1.

Tabla 1: Resumen de la revisión de literatura sobre ludificación.

\begin{tabular}{|c|c|c|}
\hline Autores & $\begin{array}{l}\text { Elementos del diseño de la } \\
\text { ludificación }\end{array}$ & Mejoras en los estudiantes \\
\hline $\begin{array}{l}\text { Abramovich Schunn y } \\
\text { Higashi (2013) }\end{array}$ & Insignias & Motivación \\
\hline $\begin{array}{l}\text { Barata, Gama, Jorge y } \\
\text { Gonçalves (2013) }\end{array}$ & $\begin{array}{l}\text { Puntos de experiencia, niveles, } \\
\text { tabla clasificatoria, retos, insignias }\end{array}$ & $\begin{array}{l}\text { Asistencia, motivación, } \\
\text { implicación, participación }\end{array}$ \\
\hline Brewer et al. (2013) & Puntos, premios & Motivación \\
\hline Coleman (2018) & Insignias & Motivación, implicación \\
\hline $\begin{array}{l}\text { de Freitas y de Freitas } \\
\text { (2013) }\end{array}$ & $\begin{array}{l}\text { Puntos de experiencia, } \\
\text { recompensas en el juego }\end{array}$ & Diversión, implicación \\
\hline Dominguez et al. (2013) & $\begin{array}{l}\text { Insignias, trofeos, barras de } \\
\text { progreso }\end{array}$ & $\begin{array}{c}\text { Motivación } \\
\text { (baja la participación) }\end{array}$ \\
\hline $\begin{array}{l}\text { Eleftheria, Charikleia, } \\
\text { lason, Athanasios y } \\
\text { Dimitrios (2013) }\end{array}$ & $\begin{array}{l}\text { Incorporación, puntos, niveles, } \\
\text { insignias, retos, repeticiones, } \\
\text { desbloquear contenidos, } \\
\text { personalización }\end{array}$ & $\begin{array}{l}\text { Experiencia de aprendizaje } \\
\text { productivo, diversión, implicación }\end{array}$ \\
\hline $\begin{array}{l}\text { Gibson, Ostashewski, } \\
\text { Flintoff, Grant y Knight } \\
\text { (2013) }\end{array}$ & Insignias & $\begin{array}{l}\text { Motivación, implicación, } \\
\text { sentimiento de logro, estatus }\end{array}$ \\
\hline Kapp (2012a) & Historia, retroalimentación & Implicación \\
\hline $\begin{array}{l}\text { Kopcha, Ding, Neumann y } \\
\text { Choi (2016) }\end{array}$ & Insignias y recompensas & Motivación \\
\hline Kumar y Khurana (2012) & Niveles, etapas, insignias, puntos & Implicación \\
\hline Lister (2015) & Puntos, insignias y obejtivos & $\begin{array}{l}\text { Motivación, asistencia y } \\
\text { participación }\end{array}$ \\
\hline $\begin{array}{l}\text { O'Donovan, Gain y Marais } \\
(2013)\end{array}$ & $\begin{array}{l}\text { Historia, elementos visuales, } \\
\text { objetivos, puntos de recompensa, } \\
\text { barra de progreso, insignias, panel } \\
\text { clasificatorio }\end{array}$ & $\begin{array}{l}\text { Implicación, motivación, } \\
\text { rendimiento }\end{array}$ \\
\hline $\begin{array}{l}\text { Ortiz-Rojas, Chiluiza y } \\
\text { Valcke (2017) }\end{array}$ & Insignias & Implicación \\
\hline $\begin{array}{l}\text { Poondej y Lerdpornkulrat } \\
\text { (2016) }\end{array}$ & $\begin{array}{l}\text { Niveles, insignias y panel } \\
\text { clasificatorio }\end{array}$ & Implicación \\
\hline $\begin{array}{l}\text { Santos, Almeida, Pedro, } \\
\text { Aresta y Koch-Grunberg } \\
(2013)\end{array}$ & Insignias & $\begin{array}{l}\text { Logro, implicación, motivación, } \\
\text { reconocimiento }\end{array}$ \\
\hline Tan y Hew (2016) & $\begin{array}{l}\text { Puntos, insignias, panel } \\
\text { clasificatorio }\end{array}$ & Motivación y participación \\
\hline Villagrasa y Duran (2013) & Historia, marcador & Implicación, motivación \\
\hline
\end{tabular}

\subsection{Elementos de la ludificación}

Esta revisión muestra los efectos positivos de la ludificación que, no obstante, dependen de la implementación de la misma (Hamari, Koivisto y Sarsa, 2014). Utilizar juegos en clase, sean clásicos, de rol o de ordenador, o utilizar sistemas de pregunta-respuesta como Kahoot no supone ludificar. La ludificación educativa requiere jugabilidad, que hace referencia al uso de elementos como puntos, insignias, tablas clasificatorias y niveles (Zichermann y Cunningham, 2011). Y aunque estos componentes ayuden a mejorar el rendimiento de los estudiantes en tareas concretas, por sí solos tampoco son suficientes (Deterding, 2014; Gåsland, 2011; Mekler, Brühlmann y Tuch, 2013). La parte menos excitante del juego son los puntos, insignias y tablas clasificatorias (Kapp, 2012b). Un buen juego tiene también retos y una historia cautivadora (Apostol, Zaharescu y Alexe, 2013; Erenli, 2013; Gåsland, 2011; Reeves y Read, 2009; Stott y Neustaedter, 2013), que aporte significación a las acciones que se les piden a los estudiantes (Kapp, 2012a). Banuri, Danková y Keefer (2017) afirman que la 
narrativa, esto es, la historia que ambienta el juego implica una mayor implicación que otros elementos como los puntos, las recompensas o los paneles de clasificación.

Ludificar no consiste en un mero añadido al currículo de elementos de juego carentes de sentido (Kapp, 2012b) dejando igual los demás elementos del proceso de enseñanzaaprendizaje. Una buena ludificación debe tener un significado especial, con capacidad de inspiración para animar al estudiante a dominar un conocimiento o competencia y dejar libertad de elección para participar (O'Donovan, Gain y Marais, 2013), por lo que requiere, como toda actividad de aprendizaje, ser diseñada de manera integrada en la programación del currículo.

\section{MÉTODO}

\subsection{Objetivos}

El objetivo central de este estudio es analizar la influencia de la ludificación en la asistencia y participación en las actividades de evaluación continua de los alumnos de estudios superiores. La ludificación no busca incidir directamente en la adquisición de aprendizajes y competencias (Huang y Soman, 2013) sino conseguirlo como consecuencia de un aumento de la implicación del alumnado en la realización de las actividades diseñadas.

\subsection{Participantes}

El muestreo utilizado ha sido el denominado de oportunidad. La muestra está compuesta por los 28 alumnos que han cursado la asignatura de Fundamentos de Diseño Instruccional y Metodologías de Aprendizaje en la especialidad de Tecnología e Informática en 2017-18, $28,6 \%$ chicas y $71,4 \%$ chicos.

\subsection{Contexto}

En la guía docente de esta asignatura del Máster en profesorado está establecido que un $30 \%$ de la calificación corresponde a la realización de tareas durante el cuatrimestre. Estas tareas tratan sobre las temáticas trabajadas durante la semana en clase y están enfocadas a promover la reflexión sobre los puntos que forman el guion de la memoria de la asignatura (40\% de la calificación). Las tareas son evaluadas por los profesores y, con el fin de aportar más puntos de vista, coevaluadas por los compañeros. La coevaluación es cualitativa.

A partir del análisis antes mencionado se introdujeron mecánicas de ludificación en los cuatro ámbitos que se quería incentivar: la asistencia, la realización correcta de las tareas, la participación en la coevaluación y la entrega puntual de las actividades de evaluación continua.

\subsection{Desarrollo}

La ludificación se realizó según el modelo Mecánicas-Dinámicas-Estética (Hunicke, LeBlanc y Zubek, 2004; Zichermann y Cunningham, 2011).

Las mecánicas definen cómo opera el juego como sistema: qué acciones se deben realizar para conseguir las insignias de los superpoderes. En este caso las acciones eran la asistencia, la entrega puntual de las tareas, la correcta consecución de las mismas y la realización de los comentarios de coevaluación a los compañeros.

Las dinámicas guían cómo los estudiantes interactúan con el juego: qué plazos de entrega y cantidad o calidad deben tener las acciones, qué insignia se consigue con cada acción y qué beneficio otorga ese superpoder. Los superpoderes se diseñaron para hacer del aprendizaje de la asignatura una experiencia gratificante. Algunos permitían acciones transgresoras, como el aplazamiento de tareas (Gestión del tiempo) o tener una segunda oportunidad para 
reelaborar y mejorar una tarea ya entregada (Aprender del error). La tabla 2 recoge las ventajas que llevaban aparejadas los superpoderes y cómo obtenerlos. Quienes al finalizar la asignatura hubieran conseguido los ocho superpoderes adquirirían el rango de Innovador intrépido y conseguirían una ventaja sorpresa en el examen de la asignatura.

La estética hace referencia a la respuesta emocional que se produce en el jugador. En este caso está ambientada por la narrativa de la consecución de superpoderes para destruir el Atontolinador. El Dr. Discriminador es el líder de la secta de los Corazones oscuros, un grupo de personas que se creen superiores a los demás. Piensan que quienes son de otra raza, país, sexo o tienen ideas distintas a las suyas son inferiores a ellos. Algunos de sus miembros como Dr. Octopus, Darth Vader o Magneto se han hecho famosos, pero la mayoría viven entre nosotros como personas de aspecto normal y no son tan fáciles de detectar. Aunque son egoístas y agresivos suelen disimularlo. Su líder, el Dr. Discriminador, ha inventado el Atontolinador, un reductor mental que, a base de ofrecer respuestas únicas y hacer creer a quien lo usa que está en posesión de la verdad y que quien no está de acuerdo con sus ideas es un ser digno de rechazo, es capaz de encoger la inteligencia y la imaginación de las personas para convertirlas en nuevos Corazones oscuros. Quien hubiera conseguido todos los superpoderes al finalizar la asignatura adquiriría el nivel de Innovador intrépido, con poder para destruir el Atontolinador.

Tabla 2: Relación de superpoderes, cómo conseguirlos y qué beneficio se obtiene

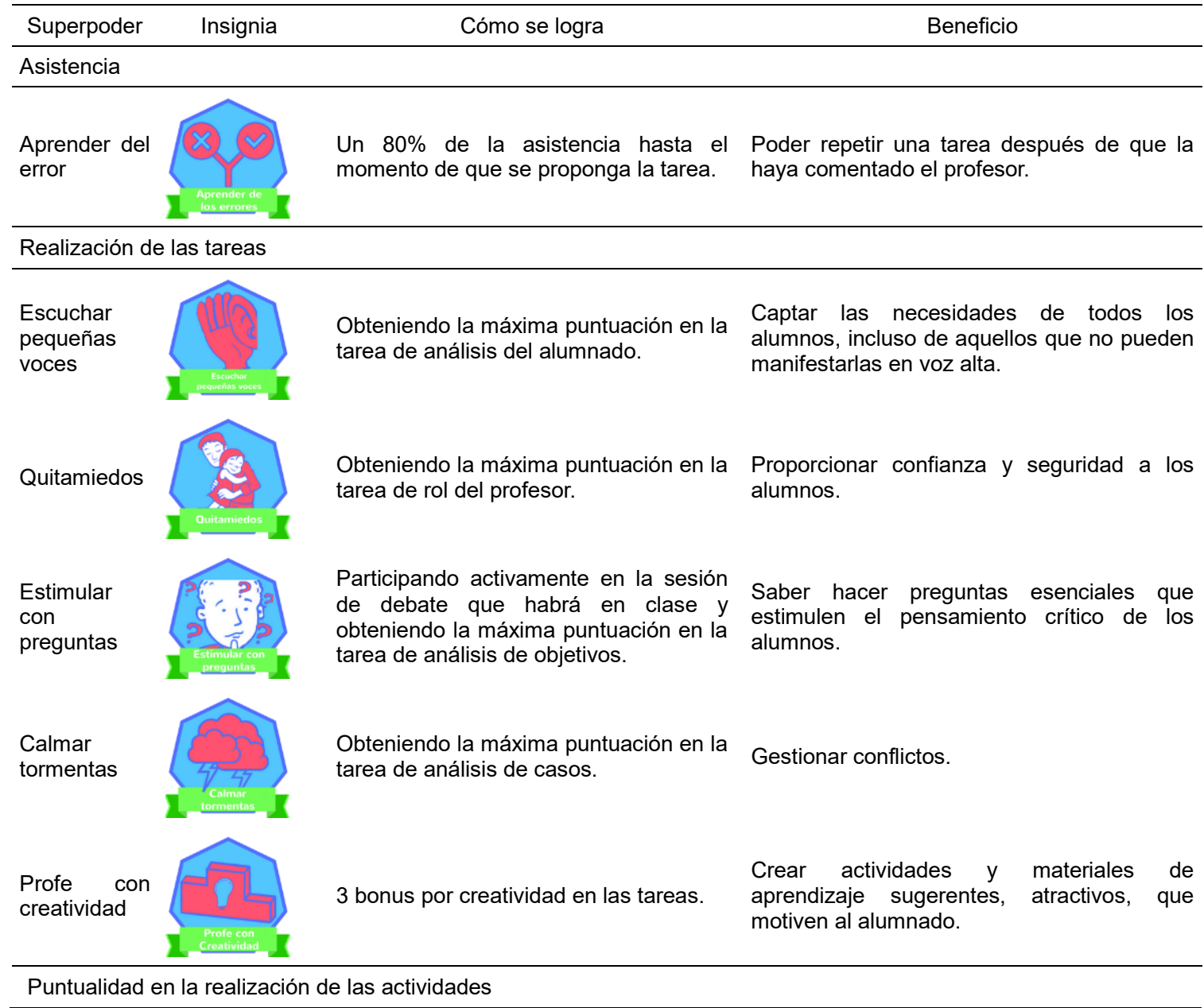

Puntualidad en la realización de las actividades 


$\begin{aligned} & \text { Gestionar el } \\ & \text { tiempo }\end{aligned}$
Realizar la coevaluación
Comunicar
se hicieron en el plazo establecido

Para visibilizar los superpoderes que cada estudiante iba consiguiendo se creó un panel con Flippity Badge Tracker en el que cada estudiante podía controlar los que ya había adquirido (imagen 1). En la wiki de la asignatura, alojada en wikispaces, se añadieron páginas con la narrativa ${ }^{2}$, con las normas y reglas del juego y con este panel.

\subsection{Instrumentos}

Para valorar los resultados se realizó un cuestionario anónimo al finalizar la asignatura y se compararon las estadísticas de participación de este curso con los de los dos años previos, no ludificados.

El cuestionario estaba estructurado en tres categorías para medir la valoración que hicieron los estudiantes (1) de la utilización de la ludificación en estudios de máster, (2) de la implementación y (3) de influencia en su actividad.

La primera sección tenía ítems para medir la motivación, basado en Hakulinen, Auvinen y Korhonen, (2013), la diversión, basado en Fitz-Walter, Tjondronegoro y Wyeth (2011) y en Denny (2013), y si era una experiencia que había merecido la pena, basado en Denny (2013) y en Domínguez et al. (2013), que habría que mantener en cursos sucesivos, basado en Hakulinen et al. (2013). Todos los ítems de esta sección se respondían con una escala Likert de 1 (completamente en desacuerdo) a 6 (completamente de acuerdo).

La segunda sección contenía ítems para que los estudiantes hicieran la valoración de la implementación. Tenía dos subsecciones. Una más específica para la narrativa, que se diseñó ad hoc para esta investigación. Constaba de una cuestión de selección múltiple donde podían marcar una o varias características: una niñería, divertida, motivadora, ambientación no adecuada a estudiantes del máster en profesorado, integrada con las capacidades que debe desarrollar un profesor/a, Otra (describir). Estas características están basadas en el análisis de los resultados del estudio de Hakulinen et al. (2013). La segunda subsección medía el grado de dificultad en la consecución de las insignias tenía un ítem que se respondía con una escala Likert de 1 (Totalmente imposible) a 6 (Demasiado fácil), basado en Li, Grossman y Fitzmaurice (2012).

La tercera sección medía la posible influencia de la ludificación en la actividad de los estudiantes, con ítems basados en Denny (2013) y en Hakulinen et al. (2013), referentes a la asistencia, la puntualidad en la entrega de las tareas y a la participación en la coevaluación. Se

\footnotetext{
2 http://desesprofesorados.wikispaces.com/El Innovador intrépido contra el Dr. Discriminador (lamentablemente está alojada en Wikispaces, que cierra el próximo 31 de julio de 2018)
} 
respondía con una escala Likert de 1 (No ha influido en absoluto) a 4 (Si no existieran no lo habría hecho).

Al final del cuestionario había una pregunta abierta para que los estudiantes hicieran una valoración global de la dinámica del Innovador intrépido contra el Dr. Discriminador.

\section{RESULTADOS}

Los datos recogidos se han analizado según las tres categorías del cuestionario: qué valoración global hacen los estudiantes de la ludificación, qué valoración hacen de la implementación llevada a cabo (historia que la ambienta y dificultad en conseguir los superpoderes) y en qué medida se ha logrado el objetivo de motivar la asistencia y la participación.

\subsection{Valoración de la ludificación}

Aunque solo el $35 \%$ de los alumnos está de acuerdo o completamente de acuerdo en considerar que la experiencia ha sido divertida (3,93 de puntuación media en una escala Likert de 1 a 6), sí que les ha resultado motivadora: el 50\% de los alumnos está de acuerdo o completamente de acuerdo en que es una dinámica que se puede valorar como tal y ninguno está en desacuerdo ni completamente en desacuerdo. La puntuación media obtenida, de 1 a 6 , es 4,4 . Además, la mayoría de los alumnos $(60 \%)$ prefiere haber aprendido en un entorno ludificado y considera que merece la pena mantenerlo para el curso que viene.

Tabla 3: Valoración global de la ludificación

Valoración media

\begin{tabular}{lc} 
& Valoración media \\
\hline Los superpoderes me han resultado motivadores & 4,42 \\
& \\
Ganar los superpoderes para conseguir ser un Innovador & 3,84 \\
intrépido ha resultado divertido & 2,05 \\
Habría preferido que no hubiera superpoderes ni Dr. & \\
Discriminador & 4,47 \\
\hline
\end{tabular}

El $78,6 \%$ consideran que las normas de participación estaban claras o muy claras $(3,14$ sobre 4 de valoración media).

Las opiniones expresadas hacen referencia a la innovación que representa en unos estudios de máster («Estrategia original de enfocar el aprendizaje de los alumnos», "Me parece una dinámica innovadora que en general hace que el alumno esté mucho más estimulado») y a que es una metodología utilizable en su futura profesión («La utilizaré», «Pienso utilizarla en el futuro (cambiando la ambientación)», «una motivación para innovar»).

\subsection{Implementación de la ludificación}

\subsubsection{Narrativa}

Al preguntarles sobre la narrativa de la lucha contra el Dr. Discriminador las valoraciones cambian: el $60 \%$ la considera divertida y el $53 \%$ motivadora. Otras calificaciones para la narrativa son "estresante", "desconcertante", "fresca" y "original". Un 33\% considera que está 
bien integrada con las capacidades que debe desarrollar un docente y solo dos alumnos (13\%) no consideran que la ambientación sea adecuada a unos estudiantes de máster.

Tabla 4: Valoración de la narrativa

La historia me ha parecido
A. Está bien integrada con las capacidades que debe desarrollar un profesor/a
$6(40 \%)$
B. Bien, pero la ambientación no resulta adecuada a estudiantes del máster en profesorado
$3(20 \%)$
C. Divertida
$11(73,3 \%)$
D. Motivadora
$10(66,7 \%)$
E. Otro
$3(20 \%)$

\subsubsection{Dificultad para conseguir los superpoderes}

Las recompensas tienen que ser deseables y alcanzables para proporcionar suficiente motivación extrínseca, pero conseguirlas tiene que suponer un reto que produzca un sentimiento de logro y satisfacción (Glover, 2013).

Una sección del cuestionario pedía a los estudiantes su valoración sobre el grado de dificultad de conseguir cada superpoder mediante una escala de Likert de 1 (totalmente imposible) a 6 (demasiado fácil). La dificultad está en un rango de 3,5 (quitamiedos) a 4,3 (aprender del error).

Tabla 5: Facilidad para conseguir los superpoderes

\begin{tabular}{lc} 
Te ha parecido fácil conseguir el superpoder ... & Valoración media \\
\hline Escuchar pequeñas voces & 3,81 \\
Quitamiedos & 3,44 \\
Estimular con preguntas & 3,5 \\
Aprender del error & 4,25 \\
Calmar tormentas & 3,5 \\
Gestionar el tiempo & 3,94 \\
Comunicar & 3,88 \\
Realizar creaciones & 3,88 \\
\hline
\end{tabular}

\subsection{Influencia en la actividad}

En esta categoría se han querido destacar las tres actividades que los profesores querían mejorar con relación a cursos anteriores: asistencia a clase, entrega puntual de las tareas y realización de comentarios de coevaluación a las tareas de los compañeros.

\subsubsection{Asistencia a clase}

Para el $53,9 \%$ de los alumnos conseguir los superpoderes no ha influido en su asistencia a clase mientras que para el $46 \%$ restante ha supuesto un incentivo en mayor o menor medida: 
el $23 \%$ lo habría hecho igual, pero se ha esmerado un poco más y para el $23 \%$ han supuesto una motivación extra.

El dato de asistencia de este curso -un $78,6 \%$ de los alumnos asistieron a clase con regularidad- muestra un incremento en relación al nivel de asistencia habitual de los cursos anteriores $(62,5 \%$ en $2015-16$ y $66,6 \%$ en $2016-17)$. Las comparaciones se recogen en el gráfico 1.

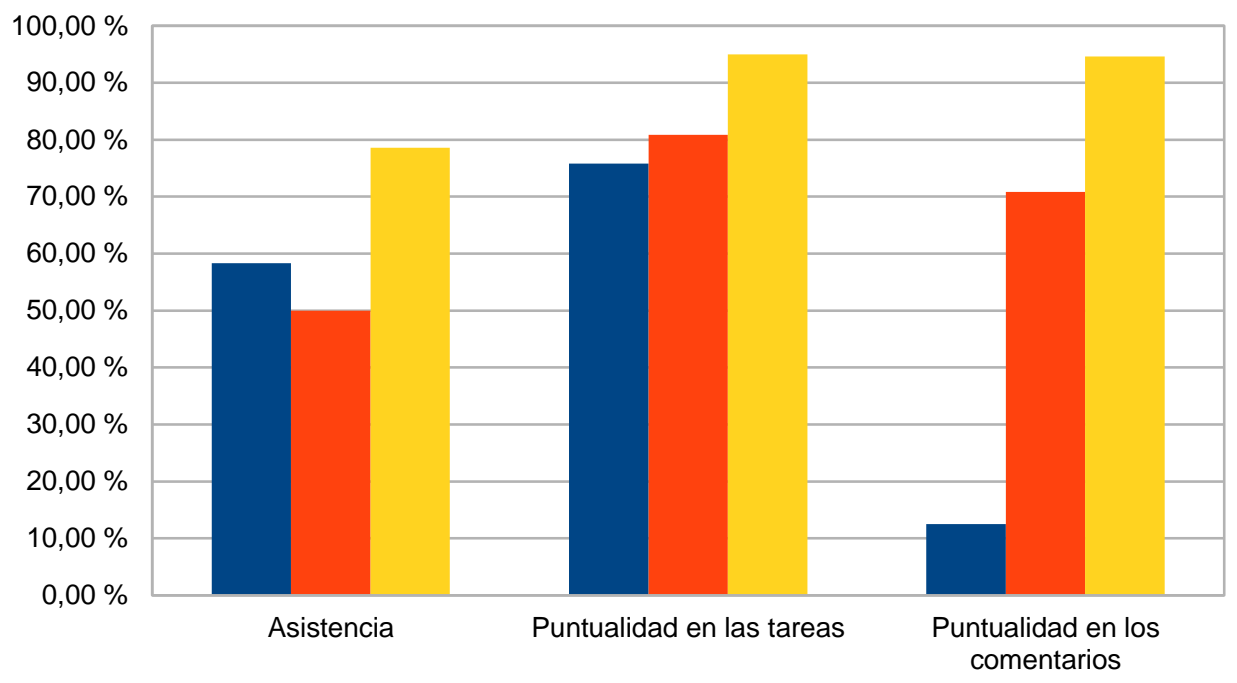

-2015-16

Gráfico 1: comparativa de realización de actividades para la evaluación continua

\subsubsection{Entrega puntual de las tareas}

Para el $38,5 \%$ de los alumnos conseguir los superpoderes no ha influido en la entrega puntual de las tareas mientras que para el $62 \%$ restante ha supuesto un incentivo en mayor 0 menor medida: el $31 \%$ lo habría hecho igual, pero se ha esmerado un poco más y para otro $31 \%$ han supuesto una motivación extra.

Ocho alumnos $(28,6 \%)$ han hecho uso en alguna ocasión del superpoder que permitía entregar la tarea con 48 horas de retraso.

La puntualidad en la entrega de tareas también se ha incrementado en relación a los cursos anteriores: Solo dos alumnos $(7,1 \%)$ entregaron tres tareas tarde y uno de ellos $(3,8 \%)$ entregó tarde o no entregó las otras dos, lo que supone un promedio del $93,8 \%$ en puntualidad, frente al $75,8 \%$ en $2015-16$, curso en el que en alguna tarea el $50 \%$ de los alumnos entregó con retraso, y un $80,8 \%$ en $2016-17$, curso en el que en una tarea el $25 \%$ de los alumnos entregó con retraso.

\subsubsection{Coevaluación de los compañeros}

Para el $31,3 \%$ de los alumnos conseguir los superpoderes no ha influido en la realización puntual de los comentarios mientras que para el $69 \%$ restante ha supuesto un incentivo en mayor o menor medida: el $50 \%$ lo habría hecho igual, pero se ha esmerado un poco más y para el $18,8 \%$ han supuesto una motivación extra.

La realización puntual de los comentarios de coevaluación a las tareas de los compañeros también se ha incrementado en relación a los cursos anteriores: Solo dos alumnos $(7,1 \%)$ dejaron en dos ocasiones de hacer los comentarios y uno de ellos $(3,8 \%)$ los hizó tarde o no los hizo tampoco en otras dos, lo que supone que el $100 \%$ han realizado los comentarios de 
coevaluación con un promedio del $94,6 \%$ en puntualidad, frente al $12,5 \%$ de participación en $2015-16^{3}$ y al $70,8 \%$ en $2016-17$.

Esta mejora en los resultados contrasta con la valoración que hacen los estudiantes de la influencia de la dinámica utilizada en su rendimiento en la asignatura, 3,9 sobre 6 de media, y la consideración de que haya sido una buena herramienta para ayudarles a llevar la asignatura al día (3,8 sobre 6$)$.

\section{DISCUSIÓN}

Los estudiantes han valorado la dinámica de los Superpoderes contra el Dr. Discriminador como motivadora (Abramovich, Schunn, y Higashi, 2013, Brewer et al., 2013, Dominguez et al., 2013, Villagrasa y Duran, 2013) y divertida (de Freitas y de Freitas, 2013).

Los resultados obtenidos demuestran que, como ya han comprobado Barata et al. (2013), Gibson et al. (2013), O'Donovan et al. (2013) y Santos et al. (2013), la ludificación mejora la implicación y la participación. El $60 \%$ de los alumnos prefiere aprender en un entorno ludificado y la dinámica ha obtenido una puntuación de 4,4 sobre 6 . La participación en las actividades de evaluación continua ha mejorado en la puntualidad en la entrega de las tareas $(93,8 \%$ en 2017 18 , frente a $75,8 \%$ en $2015-16$ y $80,8 \%$ en $2016-17$ ) y en la realización de la coevaluación a los compañeros (94,6\% en $2017-18$ frente a $12,5 \%$ de participación en $2015-16$ y 70,8\% en 2016 17). Los alumnos han reconocido que la ludificación ha influido en esta mejora (62\% en la entrega puntual de las tareas y $69 \%$ en los comentarios de coevaluación).

A ello ha contribuido que estuviera ambientada en una historia "fresca, original" (Apostol, Zaharescu y Alexe, 2013; Erenli, 2013; Gåsland, 2011; Reeves y Read, 2009; Stott y Neustaedter, 2013) que para el $80 \%$ de los alumnos daba sentido a las acciones que se les pedían (Kapp, 2012a) y que el superpoder "Aprender de los errores" contribuyó a romper la "cadena del fracaso» (Kapp, 2012b) con lo que los estudiantes, futuros docentes, pudieron ver el error como una oportunidad en lugar de sentir la presión y desaliento de fallar tras el esfuerzo en la realización de las tareas (Lee y Hammer, 2011). También ha contribuido que el grado de facilidad/dificultad para conseguir las insignias fuera el adecuado (Glover, 2013): la más fácil (Aprender del error 4,25) se lograba con un mínimo del $80 \%$ de asistencia a clase y las más difíciles (Calmar tormentas y Estimular con preguntas 3,5) se lograban realizando correctamente dos de las tareas propuestas para la evaluación continua.

Asimismo, la comparativa de los datos de asistencia con cursos anteriores y el hecho de que para un $46 \%$ del alumnado la ludificación haya supuesto, en mayor o menor medida, supone un incentivo para acudir a clase. Esto avala que la dinámica ha contribuido al objetivo de mejorarla (Barata, Gama, Jorge, y Gonçalves, 2013).

Los profesores investigadores también hemos obtenido resultados de esta primera iteración de nuestra investigación-acción, como la necesidad de que en sucesivas ediciones la narrativa de los superpoderes esté "más presente en las clases" y la posibilidad de que haya un superpoder que se puedan dar los alumnos ("me hubiera gustado poder dar un superpoder como compañera a otro.") o un superpoder "comodín".

\section{CONCLUSIONES}

Las evidencias obtenidas en esta experiencia de innovación contribuyen a aportar conocimiento sobre las ventajas del uso de la ludificación como instrumento de aprendizaje, también en la educación superior.

\footnotetext{
${ }^{3}$ Los datos de estos dos cursos indican la participación total, con o sin puntualidad
} 
Por otra parte, aportan una nueva perspectiva a las metodologías utilizables en la educación superior. La ludificación es una estrategia aplicable a cualquier área y nivel y la narrativa del Dr. Discriminador es independiente de especialidad y materia, por lo que es adaptable a cualquier grado o máster y a planes de formación del profesorado.

En esta experiencia piloto se ha conseguido una importante mejora en la participación de los alumnos en las actividades de la evaluación continua. Esta mejora ha estado influenciada por la ludificación. El alumnado la ha acogido de forma positiva, ha manifestado su preferencia por participar en la lucha contra el Dr. Discriminador y ha considerado acertado mantenerla al curso siguiente.

Los alumnos han demostrado una mayor implicación en la asignatura y han manifestado que la consecución de los superpoderes ha resultado satisfactoria y que han aprendido una metodología innovadora que les ha aportado "ideas que pueden ser aplicables en un futuro en las aulas". Han demandado poder entregar entre compañeros un superpoder a aquellos que destaquen en la realización de tareas por creatividad o profundidad.

No obstante, esta investigación presenta algunas limitaciones por el tamaño y características de la muestra, al estar compuesta por estudiantes de una única especialidad del Máster en profesorado, y porque los datos recogidos, salvo una pregunta abierta, están restringidos a respuestas cerradas. Es conveniente ampliar tanto la muestra, incluyendo a otras especialidades del máster y/o a otros estudios de máster, como la variedad de los datos, realizando entrevistas que permitan profundizar en los aspectos más reseñables de la influencia de la ludificación en la participación en clase.

\section{REFERENCIAS BIBLIOGRÁFICAS}

Abramovich, S., Schunn, C., y Higashi, R. (2013). Are badges useful in education?: It depends upon the type of badge and expertise of learner. Educational Technology Research and Development, 61(2), 217 - 232.

Apostol, S., Zaharescu, L., y Alexe, I. (2013). Gamification of learning and educational games. Paper presented at the International Scientific Conference eLearning and Software for Education, 2, 67-72.

Banuri, S., Danková, K. y Keefer, P. (2017), It's Not All Fun and Games: Feedback, Task Motivation, and Effort.

Barata, G., Gama, S., Jorge, J., y Gonçalves, D. (2013). Improving participation and learning with gamification. Proceedings of the First International Conference on gameful design, research, and applications, 10-17. doi:10.1145/2583008.2583010.

Brewer, R., Anthony, L., Brown, Q., Irwin, G., Nias, J. y Tate, B. (2013). Using Gamification to Motivate Children to Complete Empirical Studies in Lab Environments. 12th International Conference on Interaction Design and Children, pp. 388-391.

Coleman, J. D. (2018). Engaging undergraduate students in a co-curricular digital badging platform. Education and Information Technologies, pp. 1-14.

De Freitas, A.A. y de Freitas, M.M. (2013). Classroom Live: A Software-assisted Gamification Tool. Computer Science Education, vol 23(2), pp. 186-206.

Denny, P. (2013). The effect of virtual achievements on student engagement. Proceedings of the SIGCHI conference on human factors in computing systems. pp. 763-772.

Deterding, S., Dixon, D., Khaled, R., y Nacke, L. (2011). From game design elements to gamefulness: Defining "Gamification". Proceedings of the 15th International Academic MindTrek Conference, pp. 9-15. doi:10.1145/2181037.2181040 
Deterding, S. (2014). Eudaimonic Design, or: Six Invitations to Rethink Gamification. En Fuchs, M., Fizek, S., Ruffino, P. and Schrape, N. (Eds.): Rethinking Gamification. Lüneburg: meson press, pp. 305-331. Recuperado de https://www.researchgate.net/publication/263918136 Eudaimonic Design or Six Invitation $\underline{s \text { to Rethink Gamification }}$

Domínguez, A., Saenz-de-Navarrete, J., de-Marcos, L., Fernandez-Sanz, L., Pages, C. y Martinez-Herraiz, J. (2013). Gamifying learning experiences: Practical implications and outcomes. Computers y Education, 63, pp. 380-392. doi: http://dx.doi.org/10.1016/j.compedu.2012.12.020

Eleftheria, C.A., Charikleia, P., Iason, C.G., Athanasios, T. y Dimitrios, T. (2013). An Innovative Augmented Reality Educational Platform using Gamification to Enhance Lifelong Learning and Cultural Education. 4th International Conference on Information, Intelligence, Systems and Applications, pp. 1-5

Erenli, K. (2013). The Impact of Gamification - Recommending Education Scenarios. International Journal of Emerging Technologies in Learning (iJET), 8, pp. 15-21.

Fitz-Walter, Z., Tjondronegoro, D., y Wyeth, P. (2011). Orientation passport: using gamification to engage university students. In Proceedings of the 23rd Australian Computer-Human Interaction Conference, pp. 122-125.

Gåsland, M. (2011). Game mechanic based e-learning: A case study. Science and Technology, Master Thesis (June 2011). Recuperado de https://brage.bibsys.no/xmlui/handle/11250/252521

Gibson, D., Ostashewski, N., Flintoff, K., Grant, S. y Knight, E. (2013). Digital Badges in Education. Education and Information Technology. Springer, New York.

Glover, I. (2013). Play as you learn: gamification as a technique for motivating learners. En Herrington, J., Couros A. and Irivine, V. (eds.) Proceedings of World Conference on Educational Multimedia, Hypermedia and Telecommunications 2013. Chesapeake, VA, AACE, 1999-2008. Recuperado de http://shura.shu.ac.uk/7172/

Hakulinen, L., Auvinen, T. y Korhonen, A. (2013). Empirical Study on the effect of achievement badges. TRAKLA2 Online Learning Environment. Proceedings of Learning and Teaching in Computing and Engineering (LaTiCE) conference, Macau, pp. 47-54.

Hamari, J., Koivisto, J., y Sarsa, H. (2014). Does gamification work?. A literature review of empirical studies on gamification. Paper presented at the 2014 47th Hawaii International Conference on System Sciences, 3025-3034. doi:10.1109/HICSS.2014.377

Huang, W. H. Y. y Soman, D. (2013). Gamification of education. Research Report Series: Behavioural Economics in Action, Rotman School of Management, University of Toronto.

Hunicke, R., LeBlanc, M., y Zubek, R. (2004). MDA: A formal approach to game design and game research. Proceedings of the AAAl Workshop on Challenges in Game Al. 4 (1). Recuperado de https://www.cs.northwestern.edu/ hunicke/MDA.pdf

Kapp, K.M. (2012a). Games, gamification, and the quest for learner engagement. Training and Development 66(6), pp.64-68.

Kapp, K.M. (2012b). The Gamification of Learning and Instruction: Game-Based Methods and Strategies for Training and Education. San Francisco: John Wiley y Sons.

Kopcha, T. J., Ding, L., Neumann, K. L., y Choi, I. (2016). Teaching technology integration to K12 educators: A 'Gamified'approach. TechTrends, 60(1), pp. 62-69.

Kumar, B. y Khurana, P. (2012). Gamification in Education - Learn Computer Programming with Fun. International Journal of Computers and Distributed Systems 2(1), 46-53

Lee, J. J., y Hammer, J. (2011). Gamification in education: What, how, why bother? Academic exchange quarterly, 15(2), 146.1 Recuperado de https://www.uwstout.edu/soe/profdev/resources/upload/Lee-Hammer-AEQ-2011.pdf 
Li, W., Grossman, T., y Fitzmaurice, G. (2012). GamiCAD: a gamified tutorial system for first time autocad users. Proceedings of the 25th annual ACM symposium on User interface software and technology, pp. 103-112.

Lister, M. C. (2015). Gamification: The effect on student motivation and performance at the post-secondary level. Issues and Trends in Educational Technology, 3(2).

Mekler, E., Brühlmann, F., Opwis, K., y Tuch, A. (2013). Do points, levels and leaderboards harm intrinsic motivation? An empirical analysis of common gamification elements. Proceedings of the First International Conference on gameful design, research, and applications, 66-73. doi:10.1145/2583008.2583017

O'Donovan, S., Gain, J., y Marais, P. (2013). A case study in the gamification of a universitylevel games development course. Proceedings of the South African Institute for Computer Scientists and Information Technologists Conference, (SAICSIT'13), pp. 242-251. doi: $10.1145 / 2513456.2513469$

Ortiz-Rojas, M., Chiluiza, K., y Valcke, M. (2017). Gamification in Computer Programming: Effects on Learning, Engagement, Self-Efficacy and Intrinsic Motivation. In European Conference on Games Based Learning, pp. 507-514.

Poondej, C., y Lerdpornkulrat, T. (2016). The development of gamified learning activities to increase student engagement in learning. Australian Educational Computing, 31(2).

Reeves, B., y Read, J. L. (2009). Total engagement: How games and virtual worlds are changing the way people work and businesses compete. Harvard Business Press.

Sandusky, S. (2015). Gamification in education. Recuperado de https://arizona.openrepository.com/handle/10150/556222

Santos, C., Almeida, S., Pedro, L., Aresta, M. y Koch-Grunberg, T. (2013). Students' Perspectives on Badges in Educational Social Media Platforms: The Case of SAPO Campus Tutorial Badges. IEEE 13th International Conference on Advanced Learning Technologies, pp. 351-353.

Stott, A. y Neustaedter, C. (2013) Analysis of Gamification in Education, Technical Report 20130422-01, Connections Lab, Simon Fraser University, Surrey, BC, Canada, April. Recuperado de http://clab.iat.sfu.ca/pubs/Stott-Gamification.pdf

Tan, M., y Hew, K. F. (2016). Incorporating meaningful gamification in a blended learning research methods class: Examining student learning, engagement, and affective outcomes. Australasian Journal of Educational Technology, 32(5). doi: 10.14742/ajet.2232

Villagrasa, S., y Duran, J. (2013). Proceedings of the First International Conference on technological ecosystem for enhancing multiculturality, pp. 429-433. doi:10.1145/2536536.2536602

Zichermann, G., y Cunningham, C. (2011). Gamification by design: Implementing game mechanics in web and mobile apps. O'Reilly Media. 


\section{INFORMACIÓN SOBRE LOS AUTORES}

José Ma Falcó-Boudet

Universidad de Zaragoza

Es coordinador de la especialidad de Tecnología e Informática del Máster en Profesorado de Educación Secundaria de la Universidad de Zaragoza. Ha coordinado diversos proyectos de innovación docente, ha publicado varios artículos sobre el uso de las TIC en la innovación docente y ha colaborado en otros artículos técnicos.

- Twitter: @profechef

\section{José Luis Huertas Talón \\ Universidad de Zaragoza}

José Luis Huertas Talón es Catedrático de Organización y Proyectos de Fabricación Mecánica en el Centro Público Integrado de Formación Profesional "Corona de Aragón" y es Profesor Asociado en el área de Ingeniería de los procesos de fabricación de la Universidad de Zaragoza. Es el Jefe del Departamento de Innovación y Transferencia del Conocimiento del CPIFP "Corona de Aragón". Ha coordinado el proyecto "El conocimiento como motor de desarrollo de la fabricación mecánica" el cual involucró seis centros educativos de FP y siete empresas y finalizó en noviembre de 2012. Ha participado en otros proyectos de innovación y en programas de intercambio Erasmus. Es miembro del Instituto de Ingeniería de Aragón I3A dentro del grupo ID-Ergo. Ha colaborado en diversos artículos técnicos y textos

\footnotetext{
(c) (1) (2)

Los textos publicados en esta revista están sujetos a una licencia de Reconocimiento 4.0 España de Creative Commons. Puede copiarlos, distribuirlos, comunicarlos públicamente y hacer obras derivadas siempre que reconozca los créditos de las obras (autoría, nombre de la revista, institución editora) de la manera especificada por los autores o por la revista. La licencia completa se puede consultar en:Licencia Creative Commons Atribución-NoComercial-Compartir por igual 4.0 Internacional.
} 